\title{
Weighted Choquard Equation Perturbed with Weighted Nonlocal Term
}

\section{Gurpreet Singh ${ }^{1}$ (D)}

Accepted: 16 July 2021

(c) The Author(s) 2021

\section{Abstract}

We investigate the following problem

$$
-\operatorname{div}\left(v(x)|\nabla u|^{m-2} \nabla u\right)+V(x)|u|^{m-2} u=\left(|x|^{-\theta} * \frac{|u|^{b}}{|x|^{\alpha}}\right) \frac{|u|^{b-2}}{|x|^{\alpha}} u+\lambda\left(|x|^{-\gamma} * \frac{|u|^{c}}{|x|^{\beta}}\right) \frac{|u|^{c-2}}{|x|^{\beta}} u \quad \text { in } \mathbb{R}^{N},
$$

where $b, c, \alpha, \beta>0, \theta, \gamma \in(0, N), N \geq 3,2 \leq m<\infty$ and $\lambda \in \mathbb{R}$. Here, we are concerned with the existence of groundstate solutions and least energy sign-changing solutions and that will be done by using the minimization techniques on the associated Nehari manifold and the Nehari nodal set respectively.

Keywords Choquard Equation - Weighted $m$-Laplacian · Weighted nonlocal perturbation · Groundstate solution · Least energy sign-changing solutions

Mathematics Subject Classification $35 \mathrm{~A} 15 \cdot 35 \mathrm{~B} 20 \cdot 35 \mathrm{Q} 40 \cdot 35 \mathrm{Q} 75$

\section{Introduction}

In this paper, we study the problem

$$
-\operatorname{div}\left(v(x)|\nabla u|^{m-2} \nabla u\right)+V(x)|u|^{m-2} u=\left(|x|^{-\theta} * \frac{|u|^{b}}{|x|^{\alpha}}\right) \frac{|u|^{b-2}}{|x|^{\alpha}} u+\lambda\left(|x|^{-\gamma} * \frac{|u|^{c}}{|x|^{\beta}}\right) \frac{|u|^{c-2}}{|x|^{\beta}} u \quad \text { in } \mathbb{R}^{N},
$$

where $b, c, \alpha, \beta>0, \theta, \gamma \in(0, N), 2 \leq m<\infty, N \geq 3, \lambda \in \mathbb{R}$ and $\operatorname{div}\left(v(x)|\nabla u|^{m-2} \nabla u\right)$ is the weighted $m$-Laplacian. Here $v$ is a Muckenhoupt weight and $|x|^{-\xi}$ is the Riesz potential of order $\xi \in(0, N)$. The function $V \in C\left(\mathbb{R}^{N}\right)$ must satisfy either one or both of the following conditions:

(A1) $\inf _{\mathbb{R}^{N}} V(x) \geq A_{0}>0$;

(A2) For all $B>0$ the set $\left\{x \in \mathbb{R}^{N}: V(x) \leq B\right\}$ has finite Lebesgue measure.

Gurpreet Singh

gurpreet.bajwa2506@gmail.com

1 School of Mechanical and Manufacturing Engineering, Dublin City University, Dublin, Ireland 
By taking $\lambda=0$, the Eq. (1) becomes the weighted Choquard equation driven by weighted $m$-Laplacian and is given by

$$
-\operatorname{div}\left(v(x)|\nabla u|^{m-2} \nabla u\right)+V(x)|u|^{m-2} u=\left(|x|^{-\theta} * \frac{|u|^{b}}{|x|^{\alpha}}\right) \frac{|u|^{b-2}}{|x|^{\alpha}} u \quad \text { in } \mathbb{R}^{N} .
$$

The case of $v(x)=V(x) \equiv 1, m=2, \theta=b=2$ and $\alpha=0$ in (2) refers to the Choquard or nonlinear Schrödinger-Newton equation, that is,

$$
-\Delta u+u=\left(|x|^{-2} * u^{2}\right) u \quad \text { in } \mathbb{R}^{N},
$$

and it was first studied by Pekar [32] in 1954 for $N=3$. The Eq. (3) had been used by Penrose in 1996 as a model in self-gravitating matter(see [33, 34]). Also, if $v(x) \equiv 1, m=2$ and $\alpha=\lambda=0$, then (2) becomes stationary Choquard equation

$$
-\Delta u+V(x) u=\left(|x|^{-\theta} *|u|^{b}\right)|u|^{b-2} u \quad \text { in } \mathbb{R}^{N},
$$

which arises in quantum theory and in the theory of Bose-Einstein condensation. The Choquard equation has received a considerable attention in the last few decades and has been appeared in many different contexts and settings (see [1, 3, 23, 29, 31]). In [17], Du, Gao and Yang studied the following nonlinear weighted Choquard equation,

$$
-\Delta u=\frac{1}{|x|^{a}}\left(\int_{\mathbb{R}^{N}} \frac{|u(y)|^{2^{*}}}{|x-y|^{b}|y|^{c}} d y\right)|u|^{2^{*}-2} u \quad \text { in } \mathbb{R}^{N},
$$

where $N \geq 3, b \in(0, N), a \geq 0,2 a+b \leq N$ and $2^{*}=\frac{2 N-2 a-b}{N-2}$ is the critical exponent. The authors proved the existence of positive groundstate solutions by using the Schwarz symmetrization in subcritical case and by a nonlocal version of concentration-compactness principle in the critical case.

For constant weight function $v$, singular problems of the type

$$
\begin{cases}-\operatorname{div}\left(v(x)|\nabla u|^{m-2} \nabla u\right)=f(x) u^{-\delta} & \text { in } \Omega, \\ u>0 & \text { in } \Omega, \\ u=0 & \text { on } \partial \Omega,\end{cases}
$$

where $\Omega$ is a bounded smooth domain in $\mathbb{R}^{N}$ and $\delta>0$, has been considered widely in the last few decades, see [4-6,8]. The case $v \not \equiv$ constant and $m=2$ has also received a considerable attention and was considered by Hadiji and Yazidi in [24] for existence and nonexistence results, see also [21, 25]. In [9], Boccardo-Orsina studied the following singular problem

$$
\left\{\begin{aligned}
\operatorname{div}(v(x) \nabla u) & =f(x) u^{-\delta} & & \text { in } \Omega, \\
u & >0 & & \text { in } \Omega, \\
u & =0 & & \text { on } \partial \Omega,
\end{aligned}\right.
$$

where $\delta>0$ is arbitrary, $v(x)$ is a weight function satisfying $v(x) \eta \cdot \eta \geq L|\eta|^{2},|v(x)| \leq M$ for some positive constants $L, M$ and $\eta \in \mathbb{R}^{N}$. The authors were able to prove the existence of weak solution $u \in H_{0}^{1}(\Omega)$ for $0<\delta<1$ and $u \in H_{l o c}^{1}(\Omega)$ in case of $\delta>1$ such that $u^{\frac{q+1}{2}} \in H_{0}^{1}(\Omega)$. In [7], Benhamida and Yazidi investigated the critical Sobolev problem 


$$
\begin{cases}-\operatorname{div}\left(v(x)|\nabla u|^{m-2} \nabla u\right)=|u|^{b^{*}-2} u+\lambda|u|^{c-2} u & \text { in } \Omega, \\ u>0 & \text { in } \Omega, \\ u=0 & \text { on } \partial \Omega,\end{cases}
$$

where $\Omega \subset \mathbb{R}^{N}$ is a bounded domain, $N>b \geq 2, b \leq c<b^{*}$ and $b^{*}=\frac{N b}{N-b}$ is called the critical Sobolev exponent. They investigated the existence of positive solutions which depends on the weight $v(x)$. In [11], Brezis and Nirenberg studied the problem (7) for $v(x) \equiv 1$ and $m=2$ and it has stimulated a several work. In this article, we intent to choose the weight function $v$ which belongs to the class of Muckenhoupt weight $A_{p}$, see $[16,19,20]$ and this class of weights were first introduced by Muckenhoupt [30], where the author had proved that these are the only class of weights such that the Hardy-Littlewood maximal operator is bounded from the weighted Lebesgue space into itself and plays a very important role in harmonic analysis.

In the recent past, researchers are very interested in studying the problems on degenerate elliptic operators with Muckenhoupt weights. Results related to weighted Poincaré and Sobolev inequalities were obtained by Chanillo and Wheeden, see [14]. In [18], De Cicco-Vivaldi, proved a Liouville theorem for the weight $w(x)=|x|^{r}$ where $r>-N$ and $N>2$. In [26], Kawohl et al studied the related degenerate eigenvalue problem.

In this paper, we are interested in the groundstate solutions and least energy sign-changing solutions to (1) and one could easily see that (1) has a variational structure. To this aim, in the subsection below we provide variational framework and main results.

\section{Variational Framework and Main Results}

Definition 1 (Muckenhoupt Weight) Let $v \in \mathbb{R}^{N}$ be a locally integrable function such that $0<v<\infty$ a.e. in $\mathbb{R}^{N}$. Then $v \in A_{m}$, that is, the Muckenhoupt class if there exists a positive constant $C_{m, v}$ depending on $m$ and $v$ such that for all balls $B \in \mathbb{R}^{N}$, we have

$$
\left(\frac{1}{|B|} \int_{B} v d x\right)\left(\frac{1}{|B|} \int_{B} v^{-\frac{1}{m-1}} d x\right)^{m-1} \leq C_{m, v}
$$

Definition 2 (Weighted Sobolev Space) For any $v \in \mathbb{R}^{N}$, we denote the weighted Sobolev space by $W^{1, m}\left(\mathbb{R}^{N}, v\right)$ and is defined as

$$
W^{1, m}\left(\mathbb{R}^{N}, v\right)=\left\{u: \mathbb{R}^{N} \rightarrow \mathbb{R} \text { measurable }:\|u\|_{1, m, v}<\infty\right\},
$$

with respect to the norm

$$
\|u\|_{1, m, v}=\left(\int_{\mathbb{R}^{N}}|u(x)|^{m} v(x) d x+\int_{\mathbb{R}^{N}}|\nabla u|^{m} v(x) d x\right)^{\frac{1}{m}} .
$$

And the space $X=W_{0}^{1, m}\left(\mathbb{R}^{N}, v\right)$ is the closure of $\left(C_{c}^{\infty}\left(\mathbb{R}^{N}\right),\|\cdot\|_{1, m, v}\right)$ with respect to the norm

$$
\|u\|_{X}=\left(\int_{\mathbb{R}^{N}}|\nabla u|^{m} v(x) d x\right)^{\frac{1}{m}} .
$$

Definition 3 (Subclass of $A_{m}$ ) Let us denote the subclass of $A_{m}$ by $A_{p}$ and define $A_{p}$ as 


$$
A_{p}=\left\{v \in A_{m}: \quad v^{-p} \in L^{1}\left(\mathbb{R}^{N}\right) \quad \text { for some } p \in\left[\frac{1}{m-1}, \infty\right) \cap\left(\frac{N}{m}, \infty\right)\right\} .
$$

Definition 4 (Weighted Morrey space) Assume $1<m<\infty, r>0$ and $v \in A_{m}$. Then $u \in L^{m, r}\left(\mathbb{R}^{N}, v\right)$ - the weighted Morrey space, if $u \in L^{m}\left(\mathbb{R}^{N}, v\right)$, where

$$
L^{m}\left(\mathbb{R}^{N}, v\right)=\left\{u: \mathbb{R}^{N} \rightarrow \mathbb{R} \text { measurable }: \int_{\mathbb{R}^{N}} v(x)|u|^{m} d x<\infty\right\},
$$

and

$$
\|u\|_{L^{m, r}\left(\mathbb{R}^{N}, v\right)}=\sup _{x \in \mathbb{R}^{N}, R>0}\left(L \int_{B(x, R)} v(y)|u(y)|^{m} d y\right)^{\frac{1}{m}}<\infty,
$$

where $L=\frac{R^{r}}{\int_{B(x, R)} v(x) d x}$ and $B(x, R)$ is the ball centered at $x$ and radius $R$.

Next, let us define the functional space

$$
X_{v}\left(\mathbb{R}^{N}\right)=\left\{u \in X: \int_{\mathbb{R}^{N}} V(x)|u|^{m}<\infty\right\},
$$

endowed with the norm

$$
\|u\|_{X_{v}}=\left[\int_{\mathbb{R}^{N}} v(x)|\nabla u|^{m}+\int_{\mathbb{R}^{N}} V(x)|u|^{m}\right]^{\frac{1}{m}} .
$$

Also, we assume that $b$ satisfies

$$
\frac{m p(2 N-2 \alpha-\theta)}{2 N(p+1)}<b<\frac{m p(2 N-2 \alpha-\theta)}{2 N+2 p(N-m)},
$$

or

$$
\frac{2 N-2 \alpha-\theta}{2 N}<b<\infty
$$

and $c$ satisfies

$$
\frac{m p(2 N-2 \beta-\gamma)}{2 N(p+1)}<c<\frac{m p(2 N-2 \beta-\gamma)}{2 N+2 p(N-m)}
$$

or

$$
\frac{2 N-2 \beta-\gamma}{2 N}<c<\infty
$$

We also need the following double weighted Hardy-Littlewood-Sobolev inequality by Stein and Weiss(see [35])

$$
\left|\int_{\mathbb{R}^{N}}\left(|x|^{-\delta} * \frac{u}{|x|^{\mu}}\right) \frac{v}{|x|^{\mu}}\right| \leq C\|u\|_{p}\|v\|_{q},
$$

for $\delta \in(0, N), \mu \geq 0, u \in L^{p}\left(\mathbb{R}^{N}\right)$ and $v \in L^{q}\left(\mathbb{R}^{N}\right)$ such that 


$$
1-\frac{1}{q}-\frac{\delta}{N}<\frac{\mu}{N}<1-\frac{1}{q} \text { and } \frac{1}{p}+\frac{1}{q}+\frac{\delta+2 \mu}{N}=2 .
$$

Define the energy functional $\mathcal{L}_{\lambda}: X_{v}\left(\mathbb{R}^{N}\right) \rightarrow \mathbb{R}$ by

$$
\mathcal{L}_{\lambda}(u)=\frac{1}{m}\|u\|_{X_{v}}^{m}-\frac{1}{2 b} \int_{\mathbb{R}^{N}}\left(|x|^{-\theta} * \frac{|u|^{b}}{|x|^{\alpha}}\right) \frac{|u|^{b}}{|x|^{\alpha}}-\frac{\lambda}{2 c} \int_{\mathbb{R}^{N}}\left(|x|^{-\gamma} * \frac{|u|^{c}}{|x|^{\beta}}\right) \frac{|u|^{c}}{|x|^{\beta}},
$$

which is well defined by using (10) to (13) together with the double weighted Hardy-Littlewood-Sobolev inequality (14) and also $\mathcal{L}_{\lambda} \in C^{1}\left(X_{v}\right)$. Any solution of (1) is a critical point of the energy functional $\mathcal{L}_{\lambda}$. Firstly, we deal with the existence of groundstate solutions for Eq. (1). We shall be using a minimization method on the associated Nehari manifold, which is defined as

$$
\mathcal{N}_{\lambda}=\left\{u \in X_{v}\left(\mathbb{R}^{N}\right) \backslash\{0\}:\left\langle\mathcal{L}_{\lambda}^{\prime}(u), u\right\rangle=0\right\},
$$

and the groundstate solutions will be obtained as minimizers of

$$
d_{\lambda}=\inf _{u \in \mathcal{N}_{\lambda}} \mathcal{L}_{\lambda}(u)
$$

Now, we present our main result regarding the existence of groundstate solutions.

Theorem 1.1 Let $N>m \geq 2, b>c>\frac{m}{2}, \lambda>0, \theta+2 \alpha<N, \gamma+2 \beta<N$. If $b, c$ satisfies (10) and (12) or if b, c satisfy (11) and (13) and V satisfies (A1), then Eq. (1) has a groundstate solution $u \in X_{v}\left(\mathbb{R}^{N}\right)$.

Next, we study the least energy sign-changing solutions of (1). Now, we use the minimization method on the Nehari nodal set defined as

$$
\overline{\mathcal{N}}_{\lambda}=\left\{u \in X_{v}\left(\mathbb{R}^{N}\right): u^{ \pm} \neq 0 \text { and }\left\langle\mathcal{L}_{\lambda}^{\prime}(u), u^{ \pm}\right\rangle=0\right\},
$$

and solutions will be obtained as minimizers for

$$
\overline{d_{\lambda}}=\inf _{u \in \overline{\mathcal{N}}_{\lambda}} \mathcal{L}_{\lambda}(u)
$$

Here, we have

$$
\begin{aligned}
\left\langle\mathcal{L}_{\lambda}^{\prime}(u), u^{ \pm}\right\rangle= & \left\|u^{ \pm}\right\|_{X_{v}}^{m}-\int_{\mathbb{R}^{N}}\left(|x|^{-\theta} * \frac{\left(u^{ \pm}\right)^{b}}{|x|^{\alpha}}\right) \frac{\left(u^{ \pm}\right)^{b}}{|x|^{\alpha}}-\lambda \int_{\mathbb{R}^{N}}\left(|x|^{-\gamma} * \frac{\left(u^{ \pm}\right)^{c}}{|x|^{\beta}}\right) \frac{\left(u^{ \pm}\right)^{c}}{|x|^{\beta}} \\
& -\int_{\mathbb{R}^{N}}\left(|x|^{-\theta} * \frac{\left(u^{ \pm}\right)^{b}}{|x|^{\alpha}}\right) \frac{\left(u^{\mp}\right)^{b}}{|x|^{\alpha}}-\lambda \int_{\mathbb{R}^{N}}\left(|x|^{-\gamma} * \frac{\left(u^{ \pm}\right)^{c}}{|x|^{\beta}}\right) \frac{\left(u^{\mp}\right)^{c}}{|x|^{\beta}}
\end{aligned}
$$

We now state our second main result in reference to the least energy sign-changing solutions.

Theorem 1.2 Let $N>m \geq 2, b>c>m, \lambda \in \mathbb{R}, \theta+2 \alpha<m, \gamma+2 \beta<m$. If $b, c$ satisfies (10) and (12) or if b, c satisfy (11) and (13) and V satisfies both (A1) and (A2), then Eq. (1) has a least energy sign-changing solution $u \in X_{v}\left(\mathbb{R}^{N}\right)$. 
Rest of the paper is organized as follows. In Sect. 2 we collect some preliminary results. Sects. 3 and 4 consists of the proofs of our main results.

\section{Preliminary Results}

Lemma 2.1 ([2, 19, 22]) For any $v \in A_{p}$, the inclusion map

$$
X_{v} \hookrightarrow W_{0}^{1, m_{p}}\left(\mathbb{R}^{N}\right) \hookrightarrow\left\{\begin{array}{lr}
L^{s}\left(\mathbb{R}^{N}\right), & \text { for } m_{p} \leq s \leq m_{p}^{*}, \\
L^{s}\left(\mathbb{R}^{N}\right), & \text { for } 1 \leq s<\infty,
\end{array} \quad \text { when } 1 \leq m_{p}<N, m_{p}=N,\right.
$$

is continuous, where $m_{p}=\frac{m p}{p+1}$ and $m_{p}^{*}=\frac{N m_{p}}{N-m_{p}}$. Here, $m_{p}^{*}$ is called the critical Sobolev exponent. Moreover, the embeddings are compact except when $s=m_{p}^{*}$ in case of $1 \leq m_{p}<N$.

Lemma 2.2 ([27, Lemma 1.1], [28, Lemma 2.3]) There exists a constant $C_{0}>0$ such that for any $u \in X_{v}\left(\mathbb{R}^{N}\right)$ we have

$$
\int_{\mathbb{R}^{N}}|u|^{r} \leq C_{0}\|u\|\left(\sup _{y \in \mathbb{R}^{N}} \int_{B_{1}(y)}|u|^{r}\right)^{1-\frac{2}{r}},
$$

where $r \in\left[m_{p}, m_{p}^{*}\right]$.

Lemma 2.3 ([10, Proposition 4.7.12]) Let $\left(z_{n}\right)$ be a bounded sequence in $L^{r}\left(\mathbb{R}^{N}\right)$ for some $r \in(1, \infty)$ and let $\left(z_{n}\right)$ converges to $z$ almost everywhere. Then, we have that $z_{n} \rightarrow z$ weakly in $L^{r}\left(\mathbb{R}^{N}\right)$.

Lemma 2.4 (Local Brezis-Lieb lemma) Let $\left(z_{n}\right)$ be a bounded sequence in $L^{r}\left(\mathbb{R}^{N}\right)$ for some $r \in(1, \infty)$ such that $\left(z_{n}\right)$ converges to $z$ almost everywhere. Then,

$$
\left.\lim _{n \rightarrow \infty} \int_{\mathbb{R}^{N}}|| z_{n}\right|^{q}-\left|z_{n}-z\right|^{q}-\left.|z|^{q}\right|^{\frac{r}{q}}=0
$$

and

$$
\left.\lim _{n \rightarrow \infty} \int_{\mathbb{R}^{N}}|| z_{n}\right|^{q-1} z_{n}-\left|z_{n}-z\right|^{q-1}\left(z_{n}-z\right)-\left.|z|^{q-1} z\right|^{\frac{r}{q}}=0,
$$

for every $q \in[1, r]$.

Proof Let $\varepsilon>0$ be fixed, then there exists a constant $C(\varepsilon)>0$ such that

$$
|| g+\left.h\right|^{q}-\left.|g|^{q}\right|^{\frac{r}{q}} \leq \varepsilon|g|^{r}+C(\varepsilon)|h|^{r},
$$

for all $g, h \in \mathbb{R}$. By Eq. (17), we have 


$$
\begin{aligned}
\left|f_{n, \varepsilon}\right|= & \left(\left.|| z_{n}\right|^{q}-\left|z_{n}-z\right|^{q}-\left|z^{q}\right|^{\frac{r}{q}}-\varepsilon\left|z_{n}-z\right|^{r}\right)^{+} \\
& \leq(1+C(\varepsilon))|z|^{r} .
\end{aligned}
$$

Next, by Lebesgue Dominated Convergence theorem, we get

$$
\int_{\mathbb{R}^{N}} f_{n, \varepsilon} \rightarrow 0 \quad \text { as } n \rightarrow \infty .
$$

Hence, we deduce that

$$
\left.|| z_{n}\right|^{q}-\left|z_{n}-z\right|^{q}-\left.|z|^{q}\right|^{\frac{r}{q}} \leq f_{n, \varepsilon}+\varepsilon\left|z_{n}-z\right|^{r},
$$

and this further gives

$$
\left.\limsup _{n \rightarrow \infty} \int_{\mathbb{R}^{N}}|| z_{n}\right|^{q}-\left|z_{n}-z\right|^{q}-\left.|z|^{q}\right|^{\frac{r}{q}} \leq c \varepsilon,
$$

where $c=\sup _{n}\left|z_{n}-z\right|_{r}^{r}<\infty$. In order to conclude our proof, we let $\varepsilon \rightarrow 0$.

Lemma 2.5 (Weighted Nonlocal Brezis-Lieb lemma ([28, Lemma 2.4]) Let $N \geq 3$, $\alpha \geq_{2 N b}, \theta \in(0, N), \theta+2 \alpha<N$ and $b \in\left[1, \frac{2 N}{2 N-2 \alpha-\theta}\right)$. Let $\left(u_{n}\right)$ be a bounded sequence in $L \frac{-2 N b}{2 N-2 \alpha-\theta}\left(\mathbb{R}^{N}\right)$ such that $u_{n} \rightarrow$ u almost everywhere in $\mathbb{R}^{N}$. Then

$$
\int_{\mathbb{R}^{N}}\left(|x|^{-\theta} * \frac{\left|u_{n}\right|^{b}}{|x|^{\alpha}}\right) \frac{\left|u_{n}\right|^{b}}{|x|^{\alpha}} d x-\int_{\mathbb{R}^{N}}\left(|x|^{-\theta} * \frac{\left|u_{n}-u\right|^{b}}{|x|^{\alpha}}\right) \frac{\left|u_{n}-u\right|^{b}}{|x|^{\alpha}} d x \rightarrow \int_{\mathbb{R}^{N}}\left(|x|^{-\theta} * \frac{|u|^{b}}{|x|^{\alpha}}\right) \frac{|u|^{b}}{|x|^{\alpha}} d x .
$$

Proof It could be easily seen that

$$
\begin{aligned}
& \int_{\mathbb{R}^{N}}\left(|x|^{-\theta} * \frac{\left|u_{n}\right|^{b}}{|x|^{\alpha}}\right) \frac{\left|u_{n}\right|^{b}}{|x|^{\alpha}} d x-\int_{\mathbb{R}^{N}}\left(|x|^{-\theta} * \frac{\left|u_{n}-u\right|^{b}}{|x|^{\alpha}}\right) \frac{\left|u_{n}-u\right|^{b}}{|x|^{\alpha}} d x \\
& =\int_{\mathbb{R}^{N}}\left[|x|^{-\theta} *\left(\frac{1}{|x|^{\alpha}}\left|u_{n}\right|^{b}-\frac{1}{|x|^{\alpha}}\left|u_{n}-u\right|^{b}\right)\right]\left(\frac{1}{|x|^{\alpha}}\left|u_{n}\right|^{b}-\frac{1}{|x|^{\alpha}}\left|u_{n}-u\right|^{b}\right) d x \\
& +2 \int_{\mathbb{R}^{N}}\left[|x|^{-\theta} *\left(\frac{1}{|x|^{\alpha}}\left|u_{n}\right|^{b}-\frac{1}{|x|^{\alpha}}\left|u_{n}-u\right|^{b}\right)\right] \frac{1}{|x|^{\alpha}}\left|u_{n}-u\right|^{b} d x .
\end{aligned}
$$

Next, by taking $q=b, r=\frac{2 N b}{2 N-2 \alpha-\theta}$ in Lemma 2.4, we get $\left|u_{n_{2 N}}-u\right|^{b}-\left|u_{n}\right|^{b} \rightarrow|u|^{b}$ strongly in $L^{\frac{2 N}{2 N-2 \alpha-\theta}}\left(\mathbb{R}^{N}\right)$. Also, we have $\left|u_{n}-u\right|^{b} \rightarrow 0$ weakly in $L^{\frac{12 N}{2 N-2 \alpha-\theta}}\left(\mathbb{R}^{N}\right)$ by Lemma 2.3. Further, using the double weighted Hardy-Littlewood-Sobolev inequality (14) we get

$$
|x|^{-\theta} *\left(\frac{1}{|x|^{\alpha}}\left|u_{n}-u\right|^{b}-\frac{1}{|x|^{\alpha}}\left|u_{n}\right|^{b}\right) \rightarrow|x|^{-\theta} * \frac{|u|^{b}}{|x|^{\alpha}} \quad \text { in } L^{\frac{2 N}{\theta+2 \alpha}}\left(\mathbb{R}^{N}\right) .
$$

Hence, passing to the limit in (19) together with the above arguments, we get the desired result.

Lemma 2.6 Let $N \geq 3, \alpha \geq 0, \theta \in(0, N), \theta+2 \alpha<N$ and $b \in\left[1, \frac{2 N}{2 N-2 \alpha-\theta}\right)$. Assume $\left(u_{n}\right) \in L^{\frac{2 N b}{2 N-2 \alpha-\theta}}\left(\mathbb{R}^{N}\right)$ be a bounded sequence such that $u_{n} \rightarrow u$ almost everywhere in $\mathbb{R}^{N}$. Then 


$$
\int_{\mathbb{R}^{N}}\left(|x|^{-\theta} * \frac{\left|u_{n}\right|^{b}}{|x|^{\alpha}}\right) \frac{1}{|x|^{\alpha}}\left|u_{n}\right|^{b-2} u_{n} h d x \rightarrow \int_{\mathbb{R}^{N}}\left(|x|^{-\theta} * \frac{|u|^{b}}{|x|^{\alpha}}\right) \frac{1}{|x|^{\alpha}}|u|^{b-2} u h d x,
$$

for any $h \in L^{\frac{2 N b}{2 N-2 \alpha-\theta}}\left(\mathbb{R}^{N}\right)$.

Proof Let $h=h^{+}-h^{-}$and $v_{n}=u_{n}-u$. Here, it will be sufficient to prove the lemma for $h \geq 0$. One could easily notice that

$$
\begin{aligned}
\int_{\mathbb{R}^{N}}\left(|x|^{-\theta} * \frac{\left|u_{n}\right|^{b}}{|x|^{\alpha}}\right) \frac{1}{|x|^{\alpha}}\left|u_{n}\right|^{b-2} u_{n} h= & \int_{\mathbb{R}^{N}}\left[|x|^{-\theta} *\left(\frac{1}{|x|^{\alpha}}\left|u_{n}\right|^{b}-\frac{1}{|x|^{\alpha}}\left|v_{n}\right|^{b}\right)\right] \\
& \left(\frac{1}{|x|^{\alpha}}\left|u_{n}\right|^{b-2} u_{n} h-\frac{1}{|x|^{\alpha}}\left|v_{n}\right|^{b-2} v_{n} h\right) \\
+ & \int_{\mathbb{R}^{N}}\left[|x|^{-\theta} *\left(\frac{1}{|x|^{\alpha}}\left|u_{n}\right|^{b}-\frac{1}{|x|^{\alpha}}\left|v_{n}\right|^{b}\right)\right] \frac{1}{|x|^{\alpha}}\left|v_{n}\right|^{b-2} v_{n} h \\
& +\int_{\mathbb{R}^{N}}\left[|x|^{-\theta} *\left(\frac{1}{|x|^{\alpha}}\left|u_{n}\right|^{b-2} u_{n} h-\frac{1}{|x|^{\alpha}}\left|v_{n}\right|^{b-2} v_{n} h\right)\right] \frac{\left|v_{n}\right|^{b}}{|x|^{\alpha}} \\
& +\int_{\mathbb{R}^{N}}\left(|x|^{-\theta} * \frac{\left|v_{n}\right|^{b}}{|x|^{\alpha}}\right) \frac{1}{|x|^{\alpha}}\left|v_{n}\right|^{p-2} v_{n} h .
\end{aligned}
$$

Now, by taking $q=b$ and $r=\frac{2 N b}{2 N-2 \alpha-\theta}$ in Lemma 2.4 and by letting $\left(z_{n}, z\right)=\left(u_{n}, u\right)$ and then $\left(z_{n}, z\right)=\left(u_{n} h^{1 / b}, u h^{1 / b}\right)$ respectively, we get

$$
\left\{\begin{array}{l}
\frac{\left|u_{n}\right|^{b}}{|x|^{\alpha}}-\frac{\left|v_{n}\right|^{b}}{|x|^{\alpha}} \rightarrow \frac{|u|^{b}}{|x|^{\alpha}} \\
\frac{1}{|x|^{\alpha}}\left|u_{n}\right|^{b-2} u_{n} h-\frac{1}{|x|^{\alpha}}\left|v_{n}\right|^{b-2} v_{n} h \rightarrow \frac{1}{|x|^{\alpha}}|u|^{b-2} u h
\end{array} \quad \text { strongly in } L^{\frac{2 N}{2 N-2 \alpha-\theta}}\left(\mathbb{R}^{N}\right) .\right.
$$

Further, using the double weighted Hardy-Littlewood-Sobolev inequality we obtain

$$
\left\{\begin{array}{l}
|x|^{-\theta} *\left(\frac{1}{|x|^{\alpha}}\left|u_{n}\right|^{b}-\frac{1}{|x|^{\alpha}}\left|v_{n}\right|^{b}\right) \rightarrow|x|^{-\theta} * \frac{|u|^{b}}{|x|^{\alpha}} \\
|x|^{-\theta} *\left(\frac{1}{|x|^{\alpha}}\left|u_{n}\right|^{b-2} u_{n} h-\frac{1}{|x|^{\alpha}}\left|v_{n}\right|^{b-2} v_{n} h\right) \rightarrow|x|^{-\theta} *\left(\frac{1}{|x|^{\alpha}}|u|^{b-2} u h\right)
\end{array} \text { strongly in } L^{\frac{2 N}{\theta+\alpha}}\left(\mathbb{R}^{N}\right)\right.
$$

Using Lemma 2.3 we get

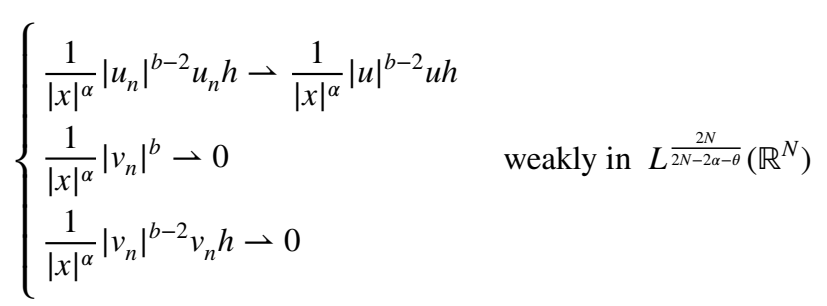

Next, by (21) and (22) we have 


$$
\begin{aligned}
& \int_{\mathbb{R}^{N}}\left[|x|^{-\theta} *\left(\frac{1}{|x|^{\alpha}}\left|u_{n}\right|^{b}-\frac{1}{|x|^{\alpha}}\left|v_{n}\right|^{b}\right)\right]\left(\frac{1}{|x|^{\alpha}}\left|u_{n}\right|^{b-2} u_{n} h-\frac{1}{|x|^{\alpha}}\left|v_{n}\right|^{b-2} v_{n} h\right) \\
& \rightarrow \int_{\mathbb{R}^{N}}\left(|x|^{-\theta} * \frac{|u|^{b}}{|x|^{\alpha}}\right) \frac{1}{|x|^{\alpha}}|u|^{b-2} u h, \\
& \int_{\mathbb{R}^{N}}\left[|x|^{-\theta} *\left(\frac{1}{|x|^{\alpha}}\left|u_{n}\right|^{b}-\frac{1}{|x|^{\alpha}}\left|v_{n}\right|^{b}\right)\right] \frac{1}{|x|^{\alpha}}\left|v_{n}\right|^{b-2} v_{n} h \rightarrow 0, \\
& \int_{\mathbb{R}^{N}}\left[|x|^{-\theta} *\left(\frac{1}{|x|^{\alpha}}\left|u_{n}\right|^{b-2} u_{n} h-\frac{1}{|x|^{\alpha}}\left|v_{n}\right|^{b-2} v_{n} h\right)\right] \frac{\left|v_{n}\right|^{b}}{|x|^{\alpha}} \rightarrow 0 .
\end{aligned}
$$

Using the double weighted Hardy-Littlewood-Sobolev inequality and Hölder's inequality, we find

$$
\begin{aligned}
\left.\left|\int_{\mathbb{R}^{N}}\left(|x|^{-\theta} * \frac{\left|v_{n}\right|^{b}}{|x|^{\alpha}}\right) \frac{1}{|x|^{\alpha}}\right| v_{n}\right|^{b-2} v_{n} h \mid & \leq\left\|v_{n}\right\|_{\frac{2 N b}{2 N-2 \alpha-\theta}}^{b}\left\|\left|v_{n}\right|^{b-1} h\right\|_{\frac{2 N}{2 N-2 \alpha-\theta}} \\
& \leq C\left\|\left|v_{n}\right|^{b-1} h\right\|_{\frac{2 N}{2 N-2 \alpha-\theta}} .
\end{aligned}
$$

Also, $v_{n}^{\frac{2 N(b-1)}{2 N-2 \alpha-\theta}} \rightarrow 0$ weakly in $L^{\frac{b}{b-1}}\left(\mathbb{R}^{N}\right)$ by Lemma 2.3. Hence,

$$
\left\|\left|v_{n}\right|^{b-1} h\right\|_{\frac{2 N}{2 N-2 \alpha-\theta}}=\left(\int_{\mathbb{R}^{N}}\left|v_{n}\right|^{\frac{2 N(b-1)}{2 N-2 \alpha-\theta}}|h|^{\frac{2 N}{2 N-2 \alpha-\theta}}\right)^{\frac{2 N-2 \alpha-\theta}{2 N}} \rightarrow 0 .
$$

Therefore, by (24) we get

$$
\lim _{n \rightarrow \infty} \int_{\mathbb{R}^{N}}\left(|x|^{-\theta} * \frac{\left|v_{n}\right|^{b}}{|x|^{\alpha}}\right) \frac{1}{|x|^{\alpha}}\left|v_{n}\right|^{b-2} v_{n} h=0,
$$

and then by passing to the limit in (20) and using (23) and (25) we conclude our proof.

In the next section, we investigate the groundstate solutions to (1).

\section{Proof of Theorem 1.1}

Proof of Theorem 1.1 depends on the analysis of the Palais-Smale sequences for $\left.\mathcal{L}_{\lambda}\right|_{\mathcal{N}_{\lambda}}$. In this section, we prove that any Palais-Smale sequence of $\left.\mathcal{L}_{\lambda}\right|_{\mathcal{N}_{\lambda}}$ is either converging strongly to its weak limit or differs from it by a finite number of sequences, which then will be the translated solutions of (2) by following the ideas from [12, 13]. Here, our approach will be depending on several weighted nonlocal Brezis-Lieb results which we have presented in Section 2. Assume $\lambda>0$. For $u, \phi \in X_{v}\left(\mathbb{R}^{N}\right)$ we have 


$$
\begin{aligned}
\left\langle\mathcal{L}_{\lambda}^{\prime}(u), \phi\right\rangle & =\int_{\mathbb{R}^{N}} v(x)|\nabla u|^{m-2} \nabla u \nabla \phi+\int_{\mathbb{R}^{N}} V(x)|u|^{m-2} u \phi-\int_{\mathbb{R}^{N}}\left(|x|^{-\theta} * \frac{|u|^{b}}{|x|^{\alpha}}\right) \frac{|u|^{b-1}}{|x|^{\alpha}} \phi \\
& -\lambda \int_{\mathbb{R}^{N}}\left(|x|^{-\gamma} * \frac{|u|^{c}}{|x|^{\beta}}\right) \frac{|u|^{c-1}}{|x|^{\beta}} \phi .
\end{aligned}
$$

Also, we have

$$
\left\langle\mathcal{L}_{\lambda}^{\prime}(t u), t u\right\rangle=t^{m}\|u\|_{X_{v}}^{m}-t^{2 b} \int_{\mathbb{R}^{N}}\left(|x|^{-\theta} * \frac{|u|^{b}}{|x|^{\alpha}}\right) \frac{|u|^{b}}{|x|^{\alpha}}-\lambda t^{2 c} \int_{\mathbb{R}^{N}}\left(|x|^{-\gamma} * \frac{|u|^{c}}{|x|^{\beta}}\right) \frac{|u|^{c}}{|x|^{\beta}},
$$

for some $t>0$.

As $b>c>\frac{m}{2}$, so the equation $\left\langle\mathcal{L}_{\lambda}^{\prime}(t u), t u\right\rangle=0$ has a unique positive solution $t=t(u)$, which is called the projection of $u$ on $\mathcal{N}_{\lambda}$. Next, we present the main properties of the Nehari manifold $\mathcal{N}_{\lambda}$ which we have used in this paper by the following lemmas:

Lemma 3.1 $\left.\mathcal{L}_{\lambda}\right|_{\mathcal{N}_{\lambda}}$ is coercive and bounded from below by a positive constant.

Proof First we show that $\left.\mathcal{L}_{\lambda}\right|_{\mathcal{N}_{\lambda}}$ is coercive. Note that

$$
\begin{aligned}
\mathcal{L}_{\lambda}(u) & =\mathcal{L}_{\lambda}(u)-\frac{1}{2 c}\left\langle\mathcal{L}_{\lambda}^{\prime}(u), u\right\rangle \\
& =\left(\frac{1}{m}-\frac{1}{2 c}\right)\|u\|_{X_{v}}^{m}+\left(\frac{1}{2 c}-\frac{1}{2 b}\right) \int_{\mathbb{R}^{N}}\left(|x|^{-\theta} * \frac{|u|^{b}}{|x|^{\alpha}}\right) \frac{|u|^{b}}{|x|^{\alpha}} \\
& \geq\left(\frac{1}{m}-\frac{1}{2 c}\right)\|u\|_{X_{v}}^{m} .
\end{aligned}
$$

Next, using the double weighted Hardy-Littlewood-Sobolev inequality together with the continuous embeddings $X_{v}\left(\mathbb{R}^{N}\right) \hookrightarrow L^{\frac{2 N b}{2 N-2 \alpha-\theta}}\left(\mathbb{R}^{N}\right)$ and $X_{v}\left(\mathbb{R}^{N}\right) \hookrightarrow L^{\frac{2 N-2 \beta-\gamma}{2 N}}\left(\mathbb{R}^{N}\right)$, for any $u \in \mathcal{N}_{\lambda}$ we have

$$
\begin{aligned}
0=\left\langle\mathcal{L}_{\lambda}^{\prime}(u), u\right\rangle & =\|u\|_{X_{v}}^{m}-\int_{\mathbb{R}^{N}}\left(|x|^{-\theta} * \frac{|u|^{b}}{|x|^{\alpha}}\right) \frac{|u|^{b}}{|x|^{\alpha}}-\lambda \int_{\mathbb{R}^{N}}\left(|x|^{-\gamma} * \frac{|u|^{c}}{|x|^{\beta}}\right) \frac{|u|^{q}}{|x|^{\beta}} \\
& \geq\|u\|_{X_{v}}^{m}-C\|u\|_{X_{v}}^{2 b}-C_{\lambda}\|u\|_{X_{v}}^{2 c} .
\end{aligned}
$$

Therefore, there exists $C_{0}>0$ such that

$$
\|u\|_{X_{v}} \geq C_{0}>0 \text { for all } u \in \mathcal{N}_{\lambda} .
$$

Hence, using coercivity of $\left.\mathcal{L}_{\lambda}\right|_{\mathcal{N}_{\lambda}}$ and (26), we get

$$
\mathcal{L}_{\lambda}(u) \geq\left(\frac{1}{m}-\frac{1}{2 c}\right) C_{0}^{m}>0 .
$$

Lemma 3.2 Let $u$ be any critical point of $\left.\mathcal{L}_{\lambda}\right|_{\mathcal{N}_{\lambda}}$. Then, it is a free critical point.

Proof Let us assume $\mathcal{K}(u)=\left\langle\mathcal{L}_{\lambda}^{\prime}(u), u\right\rangle$ for any $u \in X_{v}\left(\mathbb{R}^{N}\right)$. Using (26), for any $u \in \mathcal{N}_{\lambda}$ we get 


$$
\begin{aligned}
\left\langle\mathcal{K}^{\prime}(u), u\right\rangle & =m\|u\|^{m}-2 b \int_{\mathbb{R}^{N}}\left(|x|^{-\theta} * \frac{|u|^{b}}{|x|^{\alpha}}\right) \frac{|u|^{b}}{|x|^{\alpha}}-2 c \lambda \int_{\mathbb{R}^{N}}\left(|x|^{-\gamma} * \frac{|u|^{c}}{|x|^{\beta}}\right) \frac{|u|^{c}}{|x|^{\beta}} \\
& =(m-2 c)\|u\|_{X_{v}}^{m}-2(b-c) \int_{\mathbb{R}^{N}}\left(|x|^{-\theta} * \frac{|u|^{b}}{|x|^{\alpha}}\right) \frac{|u|^{b}}{|x|^{\alpha}} \\
& \leq-(2 c-m)\|u\|_{X_{v}}^{m} \\
& <-(2 c-m) C_{0} .
\end{aligned}
$$

Now, say $u \in \mathcal{N}_{\lambda}$ is a critical point of $\left.\mathcal{L}_{\lambda}\right|_{\mathcal{N}_{\lambda}}$. Then, by the Lagrange multiplier theorem, there exists $v \in \mathbb{R}$ such that $\mathcal{L}_{\lambda}^{\prime}(u)=v \mathcal{K}^{\prime}(u)$. Therefore, we have $\left\langle\mathcal{L}_{\lambda}^{\prime}(u), u\right\rangle=v\left\langle\mathcal{K}^{\prime}(u), u\right\rangle$. Since $\left\langle\mathcal{K}^{\prime}(u), u\right\rangle<0$, which gives us that $v=0$. Hence, $\mathcal{L}_{\lambda}^{\prime}(u)=0$.

Lemma 3.3 Any sequence $\left(u_{n}\right)$ which is a $(P S)$ sequence for $\left.\mathcal{L}_{\lambda}\right|_{\mathcal{N}_{\lambda}}$ is a $(P S)$ sequence for $\mathcal{L}_{\lambda}$.

Proof Assume that $\left(u_{n}\right) \subset \mathcal{N}_{\lambda}$ is a $(P S)$ sequence for $\left.\mathcal{L}_{\lambda}\right|_{\mathcal{N}_{\lambda}}$. As,

$$
\mathcal{L}_{\lambda}\left(u_{n}\right) \geq\left(\frac{1}{m}-\frac{1}{2 c}\right)\left\|u_{n}\right\|_{X_{v}}^{m},
$$

this gives us that $\left(u_{n}\right)$ is bounded in $X_{v}$. Next, we show that $\mathcal{L}_{\lambda}^{\prime}\left(u_{n}\right) \rightarrow 0$. Since,

$$
\mathcal{L}_{\lambda}^{\prime}\left(u_{n}\right)-v_{n} \mathcal{K}^{\prime}\left(u_{n}\right)=\left.\mathcal{L}_{\lambda}^{\prime}\right|_{\mathcal{N}_{\lambda}}\left(u_{n}\right)=o(1),
$$

for some $v_{n} \in \mathbb{R}$, we get

$$
\nu_{n}\left\langle\mathcal{K}^{\prime}\left(u_{n}\right), u_{n}\right\rangle=\left\langle\mathcal{L}_{\lambda}^{\prime}\left(u_{n}\right), u_{n}\right\rangle+o(1)=o(1) .
$$

Using (27), we have that $v_{n} \rightarrow 0$ which implies that $\mathcal{L}_{\lambda}^{\prime}\left(u_{n}\right) \rightarrow 0$.

\section{Compactness Result}

Define the energy functional $\mathcal{I}: X_{v}\left(\mathbb{R}^{N}\right) \rightarrow \mathbb{R}$ by

$$
\mathcal{I}(u)=\frac{1}{m}\|u\|^{m}-\frac{1}{2 b} \int_{\mathbb{R}^{N}}\left(|x|^{-\theta} * \frac{|u|^{b}}{|x|^{\alpha}}\right) \frac{|u|^{b}}{|x|^{\alpha}},
$$

and the associated Nehari manifold for $\mathcal{I}$ by

$$
\mathcal{N}_{\mathcal{I}}=\left\{u \in X_{v}\left(\mathbb{R}^{N}\right) \backslash\{0\}:\left\langle\mathcal{I}^{\prime}(u), u\right\rangle=0\right\},
$$

and let

$$
d_{\mathcal{I}}=\inf _{u \in \mathcal{N}_{\mathcal{I}}} \mathcal{I}(u)
$$

Also, for all $\phi \in C_{0}^{\infty}\left(\mathbb{R}^{N}\right)$, we have

$$
\left\langle\mathcal{I}^{\prime}(u), \phi\right\rangle=\int_{\mathbb{R}^{N}} v(x)|\nabla u|^{m-2} \nabla u \nabla \phi+\int_{\mathbb{R}^{N}} V(x)|u|^{m-2} u \phi-\int_{\mathbb{R}^{N}}\left(|x|^{-\theta} * \frac{|u|^{b}}{|x|^{\alpha}}\right) \frac{|u|^{b-1}}{|x|^{\alpha}} \phi .
$$

and 


$$
\left\langle\mathcal{I}^{\prime}(u), u\right\rangle=\|u\|_{X_{v}}^{m}-\int_{\mathbb{R}^{N}}\left(|x|^{-\theta} * \frac{|u|^{b}}{|x|^{\alpha}}\right) \frac{|u|^{b}}{|x|^{\alpha}} .
$$

Lemma 3.4 Let us assume that $\left(u_{n}\right) \subset \mathcal{N}_{\mathcal{I}}$ is a $(P S)$ sequence of $\left.\mathcal{L}_{\lambda}\right|_{\mathcal{N}_{\lambda}}$, that is,

(a) $\left(\mathcal{L}_{\lambda}\left(u_{n}\right)\right)$ is bounded;

(b) $\left.\mathcal{L}_{\lambda}^{\prime}\right|_{\mathcal{N}_{\lambda}}\left(u_{n}\right) \rightarrow 0$ strongly in $X_{v}^{-1}\left(\mathbb{R}^{N}\right)$.

Then there exists a solution $u \in X_{v}\left(\mathbb{R}^{N}\right)$ of (1) such that, if we replace the sequence $\left(u_{n}\right)$ with a subsequence, then one of the following alternative holds:

$\left(A_{1}\right)$ either $u_{n} \rightarrow$ u strongly in $X_{v}\left(\mathbb{R}^{N}\right)$;

or

$\left(A_{2}\right) u_{n} \rightarrow u$ weakly in $X_{v}\left(\mathbb{R}^{N}\right)$ and there exists a positive integer $k \geq 1$ and $k$ functions $u_{1}, u_{2}, \ldots, u_{k} \in X_{v}\left(\mathbb{R}^{N}\right)$ which are nontrivial weak solutions to (2) and $k$ sequences of points $\left(w_{n, 1}\right),\left(w_{n, 2}\right), \ldots,\left(w_{n, k}\right) \subset \mathbb{R}^{N}$ such that the following conditions hold:

(i) $\left|w_{n, j}\right| \rightarrow \infty$ and $\left|w_{n, j}-w_{n, i}\right| \rightarrow \infty$ if $i \neq j, n \rightarrow \infty$;

(ii) $u_{n}-\sum_{j=1}^{k} u_{j}\left(\cdot+w_{n, j}\right) \rightarrow u$ in $X_{v}\left(\mathbb{R}^{N}\right)$;

(iii) $\mathcal{L}_{\lambda}\left(u_{n}\right) \rightarrow \mathcal{L}_{\lambda}(u)+\sum_{j=1}^{k} \mathcal{I}\left(u_{j}\right)$.

Proof As $\left(u_{n}\right) \in X_{v}\left(\mathbb{R}^{N}\right)$ is a bounded sequence, so there exists $u \in X_{v}\left(\mathbb{R}^{N}\right)$ such that, up to a subsequence, we have

$$
\begin{cases}u_{n} \rightarrow u & \text { weakly in } X_{v}\left(\mathbb{R}^{N}\right), \\ u_{n} \rightarrow u & \text { weakly in } L^{S}\left(\mathbb{R}^{N}\right), m_{p} \leq s \leq m_{p}^{*}, \\ u_{n} \rightarrow u & \text { a.e. in } \mathbb{R}^{N} .\end{cases}
$$

Using (28) together with Lemma 2.6, we get

$$
\mathcal{L}_{\lambda}^{\prime}(u)=0 .
$$

Hence, $u \in X_{v}\left(\mathbb{R}^{N}\right)$ is a solution of (1). Now, if $u_{n} \rightarrow u$ strongly in $X_{v}\left(\mathbb{R}^{N}\right)$ then $\left(A_{1}\right)$ holds and we are done.

Next, let us assume that $\left(u_{n}\right) \in X_{v}\left(\mathbb{R}^{N}\right)$ does not converge strongly to $u$ and define $y_{n, 1}=u_{n}-u$. Then $\left(y_{n, 1}\right)$ converges weakly (not strongly) to zero in $X_{v}\left(\mathbb{R}^{N}\right)$ and

$$
\left\|u_{n}\right\|_{X_{v}}^{m}=\|u\|_{X_{v}}^{m}+\left\|y_{n, 1}\right\|_{X_{v}}^{m}+o(1)
$$

Using Lemma 2.5 we get

$$
\int_{\mathbb{R}^{N}}\left(|x|^{-\theta} * \frac{\left|u_{n}\right|^{b}}{|x|^{\alpha}}\right) \frac{\left|u_{n}\right|^{b}}{|x|^{\alpha}}=\int_{\mathbb{R}^{N}}\left(|x|^{-\theta} * \frac{|u|^{b}}{|x|^{\alpha}}\right) \frac{|u|^{b}}{|x|^{\alpha}}+\int_{\mathbb{R}^{N}}\left(|x|^{-\theta} * \frac{\left|y_{n, 1}\right|^{b}}{|x|^{\alpha}}\right) \frac{\left|y_{n, 1}\right|^{b}}{|x|^{\alpha}}+o(1) .
$$


By (29) and (30) we have

$$
\mathcal{L}_{\lambda}\left(u_{n}\right)=\mathcal{L}_{\lambda}(u)+\mathcal{I}\left(y_{n, 1}\right)+o(1)
$$

Now, by Lemma 2.6, for any $h \in X_{v}\left(\mathbb{R}^{N}\right)$, we have

$$
\left\langle\mathcal{I}^{\prime}\left(y_{n, 1}\right), h\right\rangle=o(1) .
$$

Further, using Lemma 2.5 we get

$$
\begin{aligned}
0=\left\langle\mathcal{L}_{\lambda}^{\prime}\left(u_{n}\right), u_{n}\right\rangle & =\left\langle\mathcal{L}_{\lambda}^{\prime}(u), u\right\rangle+\left\langle\mathcal{I}^{\prime}\left(y_{n, 1}\right), y_{n, 1}\right\rangle+o(1) \\
& =\left\langle\mathcal{I}^{\prime}\left(y_{n, 1}\right), y_{n, 1}\right\rangle+o(1)
\end{aligned}
$$

which yields

$$
\left\langle\mathcal{I}^{\prime}\left(y_{n, 1}\right), y_{n, 1}\right\rangle=o(1)
$$

Next, we claim that

$$
\Delta:=\limsup _{n \rightarrow \infty}\left(\sup _{w \in \mathbb{R}^{N}} \int_{B_{1}(w)}\left|y_{n, 1}\right|^{\frac{2 N b}{2 N-2 \alpha-\theta}}\right)>0 .
$$

Let us assume that $\Delta=0$. Using Lemma 2.2 we have $y_{n, 1} \rightarrow 0$ strongly in $L^{\frac{2 N b}{2 N-2 \alpha-\theta}}\left(\mathbb{R}^{N}\right)$. By double weighted Hardy-Littlewood-Sobolev inequality we deduce that

$$
\int_{\mathbb{R}^{N}}\left(|x|^{-\theta} * \frac{\left|y_{n, 1}\right|^{b}}{|x|^{\alpha}}\right) \frac{\left|y_{n, 1}\right|^{b}}{|x|^{\alpha}}=o(1) .
$$

Combining this together with (33), we have $y_{n, 1} \rightarrow 0$ strongly in $X_{v}\left(\mathbb{R}^{N}\right)$, which gives us a contradiction and therefore, we get $\Delta>0$.

As $\Delta>0$, one could find $w_{n, 1} \in \mathbb{R}^{N}$ such that

$$
\int_{B_{1}\left(w_{n, 1}\right)}\left|y_{n, 1}\right|^{\frac{2 N b}{2 N-2 \alpha-\theta}}>\frac{\Delta}{2} .
$$

For the sequence $\left(y_{n, 1}\left(\cdot+w_{n, 1}\right)\right)$, there exists $u_{1} \in X_{v}\left(\mathbb{R}^{N}\right)$ such that, up to a subsequence, we have

$$
\begin{array}{ll}
y_{n, 1}\left(\cdot+w_{n, 1}\right) \rightarrow u_{1} & \text { weakly in } X_{v}\left(\mathbb{R}^{N}\right), \\
y_{n, 1}\left(\cdot+w_{n, 1}\right) \rightarrow u_{1} & \text { strongly in } L_{l o c}^{\frac{2 N b}{2 N-2 \alpha-\theta}}\left(\mathbb{R}^{N}\right), \\
y_{n, 1}\left(\cdot+w_{n, 1}\right) \rightarrow u_{1} & \text { a.e. in } \mathbb{R}^{N} .
\end{array}
$$

By passing to the limit in (34), we have

$$
\int_{B_{1}(0)}\left|u_{1}\right|^{\frac{2 N b}{2 N-2 \alpha-\theta}} \geq \frac{\Delta}{2},
$$

hence, $u_{1} \not \equiv 0$. As $\left(y_{n, 1}\right)$ converges weakly to zero in $X_{v}\left(\mathbb{R}^{N}\right)$, we get that $\left(w_{n, 1}\right)$ is unbounded. Therefore, passing to a subsequence, one could assume that $\left|w_{n, 1}\right| \rightarrow \infty$. Using (33), we have $\mathcal{I}^{\prime}\left(u_{1}\right)=0$, which further implies that $u_{1}$ is a nontrivial solution of (2). Now, we define 


$$
y_{n, 2}(x)=y_{n, 1}(x)-u_{1}\left(x-w_{n, 1}\right) .
$$

Then, following the same procedure as before, we get

$$
\left\|y_{n, 1}\right\|^{m}=\left\|u_{1}\right\|^{m}+\left\|y_{n, 2}\right\|^{m}+o(1) .
$$

By Lemma 2.5 we have

$$
\int_{\mathbb{R}^{N}}\left(|x|^{-\theta} * \frac{\left|y_{n, 1}\right|^{b}}{|x|^{\alpha}}\right) \frac{\left|y_{n, 1}\right|^{b}}{|x|^{\alpha}}=\int_{\mathbb{R}^{N}}\left(|x|^{-\theta} * \frac{\left|u_{1}\right|^{b}}{|x|^{\alpha}}\right) \frac{\left|u_{1}\right|^{b}}{|x|^{\alpha}}+\int_{\mathbb{R}^{N}}\left(|x|^{-\theta} * \frac{\left|y_{n, 2}\right|^{b}}{|x|^{\alpha}}\right) \frac{\left|y_{n, 2}\right|^{b}}{|x|^{\alpha}}+o(1) .
$$

Therefore,

$$
\mathcal{I}\left(y_{n, 1}\right)=\mathcal{I}\left(u_{1}\right)+\mathcal{I}\left(y_{n, 2}\right)+o(1) .
$$

Using (31), we have

$$
\mathcal{L}_{\lambda}\left(u_{n}\right)=\mathcal{L}_{\lambda}(u)+\mathcal{I}\left(u_{1}\right)+\mathcal{I}\left(y_{n, 2}\right)+o(1) .
$$

Using the same approach as above, we get

$$
\left\langle\mathcal{I}^{\prime}\left(y_{n, 2}\right), h\right\rangle=o(1) \quad \text { for any } h \in X_{v}\left(\mathbb{R}^{N}\right)
$$

and

$$
\left\langle\mathcal{I}^{\prime}\left(y_{n, 2}\right), y_{n, 2}\right\rangle=o(1) .
$$

Further, if $\left(y_{n, 2}\right) \rightarrow 0$ strongly, then we are done by taking $k=1$ in the Lemma 3.4. Assume $y_{n, 2} \rightarrow 0$ weakly (not strongly) in $X_{v}\left(\mathbb{R}^{N}\right)$, then we could iterate the whole process and in $k$ number of steps we find a set of sequences $\left(w_{n, j}\right) \subset \mathbb{R}^{N}, 1 \leq j \leq k$ with

$$
\left|w_{n, j}\right| \rightarrow \infty \quad \text { and } \quad\left|w_{n, i}-w_{n, j}\right| \rightarrow \infty \quad \text { as } n \rightarrow \infty, i \neq j
$$

and $k$ nontrivial solutions $u_{1}, u_{2}, \cdots, u_{k} \in X_{v}\left(\mathbb{R}^{N}\right)$ of (2) such that, by denoting

$$
y_{n, j}(x):=y_{n, j-1}(x)-u_{j-1}\left(x-w_{n, j-1}\right), \quad 2 \leq j \leq k,
$$

we get

$$
y_{n, j}\left(x+w_{n, j}\right) \rightarrow u_{j} \quad \text { weakly in } X_{v}\left(\mathbb{R}^{N}\right)
$$

and

$$
\mathcal{L}_{\lambda}\left(u_{n}\right)=\mathcal{L}_{\lambda}(u)+\sum_{j=1}^{k} \mathcal{I}\left(u_{j}\right)+\mathcal{I}\left(y_{n, k}\right)+o(1) .
$$

Now, as $\mathcal{L}_{\lambda}\left(u_{n}\right)$ is bounded and $\mathcal{I}\left(u_{j}\right) \geq d_{\mathcal{I}}$, one could iterate the process only a finite number of times and with this, we conclude our proof.

Corollary 1 Any $(P S)_{c}$ sequence of $\left.\mathcal{L}_{\lambda}\right|_{\mathcal{N}_{\lambda}}$ is relatively compact for any $c \in\left(0, d_{\mathcal{I}}\right)$. 
Proof Let us assume that $\left(u_{n}\right)$ is a $(P S)_{c}$ sequence of $\left.\mathcal{L}_{\lambda}\right|_{\mathcal{N}}$. Then, by Lemma 3.4 we get $\mathcal{I}\left(u_{j}\right) \geq d_{\mathcal{I}}$ and upto a subsequence $u_{n} \rightarrow u$ strongly in $X_{v}\left(\mathbb{R}^{N}\right)$ and hence, $u$ is a solution of (1).

\section{Completion of the Proof of Theorem 1.1}

We need the following result in order to complete the proof of Theorem 1.1.

\section{Lemma 3.5}

$$
d_{\lambda}<d_{\mathcal{I}}
$$

Proof Let us assume that $P \in X_{v}\left(\mathbb{R}^{N}\right)$ is a groundstate solution of $(2)$ (see $\left.[8,15]\right)$. Let us denote by $t P$, the projection of $P$ on $\mathcal{N}_{\lambda}$, that is, $t=t(P)>0$ is the unique real number such that $t P \in \mathcal{N}_{\lambda}$. Since, $P \in \mathcal{N}_{\mathcal{I}}$ and $t P \in \mathcal{N}_{\lambda}$, we have

$$
\|P\|^{m}=\int_{\mathbb{R}^{N}}\left(|x|^{-\theta} * \frac{|P|^{b}}{|x|^{\alpha}}\right) \frac{|P|^{b}}{|x|^{\alpha}}
$$

and

$$
t^{m}\|P\|^{m}=t^{2 b} \int_{\mathbb{R}^{N}}\left(|x|^{-\theta} * \frac{|P|^{b}}{|x|^{\alpha}}\right) \frac{|P|^{b}}{|x|^{\alpha}}+\lambda t^{2 c} \int_{\mathbb{R}^{N}}\left(|x|^{-\gamma} * \frac{|P|^{c}}{|x|^{\beta}}\right) \frac{|P|^{c}}{|x|^{\beta}} .
$$

Therefore, we get $t<1$. Now,

$$
\begin{aligned}
d_{\lambda} \leq \mathcal{L}_{\lambda}(t P) & =\frac{1}{m} t^{m}\|P\|^{m}-\frac{1}{2 b} t^{2 b} \int_{\mathbb{R}^{N}}\left(|x|^{-\theta} * \frac{|P|^{b}}{|x|^{\alpha}}\right) \frac{|P|^{b}}{|x|^{\alpha}}-\frac{\lambda}{2 c} t^{2 c} \int_{\mathbb{R}^{N}}\left(|x|^{-\gamma} * \frac{|P|^{c}}{|x|^{\beta}}\right) \frac{|P|^{c}}{|x|^{\beta}} \\
& =\left(\frac{t^{m}}{m}-\frac{t^{2 b}}{2 b}\right)\|P\|^{m}-\frac{1}{2 c}\left(t^{m}\|P\|^{m}-t^{2 b} \int_{\mathbb{R}^{N}}\left(|x|^{-\theta} * \frac{|P|^{b}}{|x|^{\alpha}}\right) \frac{|P|^{b}}{|x|^{\alpha}}\right) \\
& =t^{m}\left(\frac{1}{m}-\frac{1}{2 c}\right)\|P\|^{m}+t^{2 b}\left(\frac{1}{2 c}-\frac{1}{2 b}\right)\|P\|^{m} \\
& <\left(\frac{1}{m}-\frac{1}{2 c}\right)\|P\|^{m}+\left(\frac{1}{2 c}-\frac{1}{2 b}\right)\|P\|^{m} \\
& <\left(\frac{1}{m}-\frac{1}{2 b}\right)\|P\|^{m}=\mathcal{I}(P)=d_{\mathcal{I}} .
\end{aligned}
$$

Hence, we are done.

Next, we use the Ekeland variational principle, that is, for any $n \geq 1$ there exists $\left(u_{n}\right) \in \mathcal{N}_{\lambda}$ such that

$$
\begin{array}{lrl}
\mathcal{L}_{\lambda}\left(u_{n}\right) \leq d_{\lambda}+\frac{1}{n} & \text { for all } n \geq 1, \\
\mathcal{L}_{\lambda}\left(u_{n}\right) \leq \mathcal{L}_{\lambda}(\tilde{u})+\frac{1}{n}\left\|\tilde{u}-u_{n}\right\| & \text { for all } \tilde{u} \in \mathcal{N}_{\lambda}, n \geq 1 .
\end{array}
$$

Further, one could easily find that $\left(u_{n}\right) \in \mathcal{N}_{\lambda}$ is a $(P S)_{d_{\lambda}}$ sequence for $\mathcal{L}_{\lambda}$ on $\mathcal{N}_{\lambda}$. Then, by Lemma 3.5 and Corollary 1 we have that up to a subsequence $u_{n} \rightarrow u$ strongly in $X_{v}\left(\mathbb{R}^{N}\right)$ which is a groundstate solution of the $\mathcal{L}_{\lambda}$. 


\section{Proof of Theorem 1.2}

In this section, we are concerned the existence of a least energy sign-changing solution of (1).

\section{Proof of Theorem}

Lemma 4.1 Let $N>m \geq 2, \quad b>c>m$ and $\lambda \in \mathbb{R}$. There exists a unique pair $\left(\tau_{0}, \delta_{0}\right) \in(0, \infty) \times(0, \infty)$ such that, for any $u \in X_{v}\left(\mathbb{R}^{N}\right)$ and $u^{ \pm} \neq 0$, we have $\tau_{0} u^{+}+\delta_{0} u^{-} \in \overline{\mathcal{N}}_{\lambda}$. Also, if $u \in \overline{\mathcal{N}}_{\lambda}$ then for all $\tau, \delta \geq 0$ we have $\mathcal{L}_{\lambda}(u) \geq \mathcal{L}_{\lambda}\left(\tau u^{+}+\delta u^{-}\right)$.

Proof In order to prove this lemma, we follow the idea of [36]. Define the function $\varphi:[0, \infty) \times[0, \infty) \rightarrow \mathbb{R}$ by

$$
\begin{aligned}
\varphi(\tau, \delta)= & \mathcal{L}_{\lambda}\left(\tau^{\frac{1}{2 b}} u^{+}+\delta^{\frac{1}{2 b}} u^{-}\right) \\
= & \frac{\tau^{\frac{m}{2 b}}}{m}\left\|u^{+}\right\|_{X_{v}}^{m}+\frac{\delta^{\frac{m}{2 b}}}{m}\left\|u^{-}\right\|_{X_{v}}^{m}-\lambda \frac{\tau^{\frac{c}{b}}}{2 c} \int_{\mathbb{R}^{N}}\left(|x|^{-\gamma} * \frac{\left(u^{+}\right)^{c}}{|x|^{\beta}}\right) \frac{\left(u^{+}\right)^{c}}{|x|^{\beta}}-\lambda \frac{\delta^{\frac{c}{b}}}{2 c} \\
& \int_{\mathbb{R}^{N}}\left(|x|^{-\gamma} * \frac{\left(u^{-}\right)^{c}}{|x|^{\beta}}\right) \frac{\left(u^{-}\right)^{c}}{|x|^{\beta}} \\
& -\lambda \frac{\tau \frac{c}{\frac{2 b}{2 b}}}{2 c} \int_{\mathbb{R}^{N}}\left(|x|^{-\gamma} * \frac{\left(u^{+}\right)^{c}}{|x|^{\beta}}\right) \frac{\left(u^{-}\right)^{c}}{|x|^{\beta}}-\frac{\tau}{2 b} \int_{\mathbb{R}^{N}}\left(|x|^{-\theta} * \frac{\left(u^{+}\right)^{b}}{|x|^{\alpha}}\right) \frac{\left(u^{+}\right)^{b}}{|x|^{\alpha}} \\
& -\frac{\delta}{2 b} \int_{\mathbb{R}^{N}}\left(|x|^{-\theta} * \frac{\left(u^{-}\right)^{b}}{|x|^{\alpha}}\right) \frac{\left(u^{-}\right)^{b}}{|x|^{\alpha}}-\frac{\tau^{\frac{1}{2}} \delta^{\frac{1}{2}}}{2 b} \int_{\mathbb{R}^{N}}\left(|x|^{-\theta} * \frac{\left(u^{+}\right)^{b}}{|x|^{\alpha}}\right) \frac{\left(u^{-}\right)^{b}}{|x|^{\alpha}}
\end{aligned}
$$

One could observe that $\varphi$ is strictly concave. Hence, $\varphi$ has at most one maximum point. On the other hand we have

$$
\lim _{\tau \rightarrow \infty} \varphi(\tau, \delta)=-\infty \text { for all } \delta \geq 0 \quad \text { and } \quad \lim _{\delta \rightarrow \infty} \varphi(\tau, \delta)=-\infty \text { for all } \tau \geq 0,
$$

and it could be easily seen that

$$
\lim _{\tau \searrow 0} \frac{\partial \varphi}{\partial \tau}(\tau, \delta)=\infty \text { for all } \delta>0 \quad \text { and } \lim _{\delta \searrow 0} \frac{\partial \varphi}{\partial \delta}(\tau, \delta)=\infty \text { for all } \tau>0 .
$$

Therefore, by (36) and (37) maximum cannot be achieved at the boundary. Hence, $\varphi$ has exactly one maximum point $\left(\tau_{0}, \delta_{0}\right) \in(0, \infty) \times(0, \infty)$.

Next, we divide our proof into two steps.

Step 1 .The energy level $\overline{d_{\lambda}} \geq 0$ is achieved by some $\sigma \in \overline{\mathcal{N}}_{\lambda}$.

Let us assume that $\left(u_{n}\right) \subset \overline{\mathcal{N}}_{\lambda}$ be a minimizing sequence for $\overline{d_{\lambda}}$. Observe that 


$$
\begin{aligned}
\mathcal{L}_{\lambda}\left(u_{n}\right) & =\mathcal{L}_{\lambda}\left(u_{n}\right)-\frac{1}{2 c}\left\langle\mathcal{L}_{\lambda}^{\prime}\left(u_{n}\right), u_{n}\right\rangle \\
& =\left(\frac{1}{m}-\frac{1}{2 c}\right)\left\|u_{n}\right\|_{X_{v}}^{m}+\left(\frac{1}{2 c}-\frac{1}{2 b}\right) \int_{\mathbb{R}^{N}}\left(|x|^{-\theta} * \frac{|u|^{b}}{|x|^{\alpha}}\right) \frac{|u|^{b}}{|x|^{\alpha}} \\
& \geq\left(\frac{1}{m}-\frac{1}{2 c}\right)\left\|u_{n}\right\|_{X_{v}}^{m} \\
& \geq C\left\|u_{n}\right\|_{X_{v}}^{m},
\end{aligned}
$$

for some positive constant $C_{1}>0$. Hence, for $C_{2}>0$ we have

$$
\left\|u_{n}\right\|_{X_{v}}^{m} \leq C_{2} \mathcal{L}_{\lambda}\left(u_{n}\right) \leq M
$$

that is, $\left(u_{n}\right)$ is bounded in $X_{v}\left(\mathbb{R}^{N}\right)$. This further implies that $\left(u_{n}^{+}\right)$and $\left(u_{n}^{-}\right)$are also bounded in $X_{v}\left(\mathbb{R}^{N}\right)$. Therefore, passing to a subsequence, there exists $u^{+}, u^{-} \in X_{v}\left(\mathbb{R}^{N}\right)$ such that

$$
u_{n}^{+} \rightarrow u^{+} \text {and } u_{n}^{-} \rightarrow u^{-} \quad \text { weakly in } X_{v}\left(\mathbb{R}^{N}\right) .
$$

As $b, c>m \geq_{2 N b} 2$ satisfy (10) and (12) or $_{N_{c}}(11)$ and (13), we have that the embeddings $X_{v}\left(\mathbb{R}^{N}\right) \hookrightarrow L^{\frac{2 N b}{2 N-2 \alpha-\theta}}\left(\mathbb{R}^{N}\right)$ and $X_{v}\left(\mathbb{R}^{N}\right) \hookrightarrow L^{\frac{2 N c}{2 N-2 \beta-\gamma}}\left(\mathbb{R}^{N}\right)$ are compact. Hence,

$$
u_{n}^{ \pm} \rightarrow u^{ \pm} \quad \text { strongly in } L^{\frac{2 N b}{2 N-2 \alpha-\theta}}\left(\mathbb{R}^{N}\right) \cap L^{\frac{2 N c}{2 N-2 \beta-\gamma}}\left(\mathbb{R}^{N}\right) .
$$

Using the double weighted Hardy-Littlewood-Sobolev inequality, we have

$$
\begin{aligned}
C\left(\left\|u_{n}^{ \pm}\right\|_{L_{\frac{2 N b}{2 N-2 \alpha-\theta}}^{m}}^{m}+\left\|u_{n}^{ \pm}\right\|_{L^{\frac{2 N c}{2 N-2 \beta-\gamma}}}^{m}\right) \leq & \left\|u_{n}^{ \pm}\right\|_{X_{v}}^{m} \\
= & \int_{\mathbb{R}^{N}}\left(|x|^{-\theta} * \frac{\left|u_{n}\right|^{b}}{|x|^{\alpha}}\right) \frac{\left|u_{n}^{ \pm}\right|^{b}}{|x|^{\alpha}} \\
& +|\lambda| \int_{\mathbb{R}^{N}}\left(|x|^{-\gamma} * \frac{\left|u_{n}\right|^{c}}{|x|^{\beta}}\right) \frac{\left|u_{n}^{ \pm}\right|^{c}}{|x|^{\beta}} \\
\leq & C\left(\left\|u_{n}^{ \pm}\right\|_{L^{\frac{2 N b}{2 N-2 \alpha-\theta}}}^{b}+\left\|u_{n}^{ \pm}\right\|_{L^{\frac{2 N c}{2 N-2 \beta-\gamma}}}^{c}\right) \\
\leq & C\left(\left\|u_{n}^{ \pm}\right\|_{L^{\frac{2 N b}{2 N-2 \alpha-\theta}}}^{m}+\left\|u_{n}^{ \pm}\right\|_{L^{\frac{2 N c}{2 N-2 \beta-\gamma}}}^{m}\right) \\
& \left(\left\|u_{n}^{ \pm}\right\|_{L_{\frac{2 N b}{2 N-2 \alpha-\theta}}^{b-m}+\left\|u_{n}^{ \pm} \mid\right\|_{L^{\frac{2 N c}{2 N-2 \beta-\gamma}}}^{c-m}}^{b}\right) .
\end{aligned}
$$

As $u_{n}^{ \pm} \neq 0$, we get

$$
\left\|u_{n}^{ \pm}\right\|_{L^{\frac{2 N b}{2 N-2 \alpha-\theta}}}^{b-m}+\left\|u_{n}^{ \pm}\right\|_{L^{\frac{2 N c}{2 N-2 \beta-\gamma}}}^{c-m} \geq C>0 \quad \text { for all } n \geq 1
$$

Therefore, using (38) and (39) one could have that $u^{ \pm} \neq 0$. Next, using (38) together with double weighted Hardy-Littlewood-Sobolev inequality, we deduce 


$$
\begin{aligned}
& \int_{\mathbb{R}^{N}}\left(|x|^{-\theta} * \frac{\left(u_{n}^{ \pm}\right)^{b}}{|x|^{\alpha}}\right) \frac{\left(u_{n}^{ \pm}\right)^{b}}{|x|^{\alpha}} \rightarrow \int_{\mathbb{R}^{N}}\left(|x|^{-\theta} * \frac{\left(u^{ \pm}\right)^{b}}{|x|^{\alpha}}\right) \frac{\left(u^{ \pm}\right)^{b}}{|x|^{\alpha}} \\
& \int_{\mathbb{R}^{N}}\left(|x|^{-\theta} * \frac{\left(u_{n}^{+}\right)^{b}}{|x|^{\alpha}}\right) \frac{\left(u_{n}^{-}\right)^{b}}{|x|^{\alpha}} \rightarrow \int_{\mathbb{R}^{N}}\left(|x|^{-\theta} * \frac{\left(u^{+}\right)^{b}}{|x|^{\alpha}}\right) \frac{\left(u^{-}\right)^{b}}{|x|^{\alpha}} \\
& \int_{\mathbb{R}^{N}}\left(|x|^{-\gamma} * \frac{\left(u_{n}^{ \pm}\right)^{c}}{|x|^{\beta}}\right) \frac{\left(u_{n}^{ \pm}\right)^{c}}{|x|^{\beta}} \rightarrow \int_{\mathbb{R}^{N}}\left(|x|^{-\gamma} * \frac{\left(u^{ \pm}\right)^{c}}{|x|^{\beta}}\right) \frac{\left(u^{ \pm}\right)^{c}}{|x|^{\beta}}
\end{aligned}
$$

and

$$
\int_{\mathbb{R}^{N}}\left(|x|^{-\gamma} * \frac{\left(u_{n}^{+}\right)^{c}}{|x|^{\beta}}\right) \frac{\left(u_{n}^{-}\right)^{c}}{|x|^{\beta}} \rightarrow \int_{\mathbb{R}^{N}}\left(|x|^{-\gamma} * \frac{\left(u^{+}\right)^{c}}{|x|^{\beta}}\right) \frac{\left(u^{-}\right)^{c}}{|x|^{\beta}} .
$$

Next, by using Lemma 4.1 , we get that there exists a unique pair $\left(\tau_{0}, \delta_{0}\right)$ such that $\tau_{0} u^{+}+\delta_{0} u^{-} \in \overline{\mathcal{N}}_{\lambda}$. Further, using the fact that the norm $\|.\|_{X_{v}}$ is weakly lower semi-continuous, we get

$$
\begin{aligned}
\overline{d_{\lambda}} \leq \mathcal{L}_{\lambda}\left(\tau_{0} u^{+}+\delta_{0} u^{-}\right) & \leq \liminf _{n \rightarrow \infty} \mathcal{L}_{\lambda}\left(\tau_{0} u^{+}+\delta_{0} u^{-}\right) \\
& \leq \limsup _{n \rightarrow \infty} \mathcal{L}_{\lambda}\left(\tau_{0} u^{+}+\delta_{0} u^{-}\right) \\
& \leq \lim _{n \rightarrow \infty} \mathcal{L}_{\lambda}\left(u_{n}\right) \\
& =\overline{d_{\lambda}} .
\end{aligned}
$$

We conclude by taking $\sigma=\tau_{0} u^{+}+\delta_{0} u^{-} \in \overline{\mathcal{N}}_{\lambda}$.

Step 2. $\mathcal{L}_{\lambda}^{\prime}(\sigma)=0$, that is, $\sigma \in \overline{\mathcal{N}}_{\lambda}$ is the critical point of $\mathcal{L}_{\lambda}: X_{v}\left(\mathbb{R}^{N}\right) \rightarrow \mathbb{R}$.

Say $\sigma$ is not a critical point of $\mathcal{L}_{\lambda}$, then there exists $\kappa \in C_{c}^{\infty}\left(\mathbb{R}^{N}\right)$ such that $\left\langle\mathcal{L}_{\lambda}^{\prime}(\sigma), \kappa\right\rangle=-2$. As $\mathcal{L}_{\lambda}$ is continuous and differentiable, so there exists $\zeta>0$ small such that

$$
\left\langle\mathcal{L}_{\lambda}^{\prime}\left(\tau u^{+}+\delta u^{-}+\omega \bar{\sigma}\right), \bar{\sigma}\right\rangle \leq-1 \quad \text { if }\left(\tau-\tau_{0}\right)^{2}+\left(\delta-\delta_{0}\right)^{2} \leq \zeta^{2} \text { and } 0 \leq \omega \leq \zeta
$$

Next, let us asumme that $D \subset \mathbb{R}^{2}$ is an open disc of radius $\zeta>0$ centered at $\left(\tau_{0}, \delta_{0}\right)$ and define a continuous function $\Phi: D \rightarrow[0,1]$ by

$$
\Phi(\tau, \delta)= \begin{cases}1 & \text { if }\left(\tau-\tau_{0}\right)^{2}+\left(\delta-\delta_{0}\right)^{2} \leq \frac{\zeta^{2}}{16} \\ 0 & \text { if }\left(\tau-\tau_{0}\right)^{2}+\left(\delta-\delta_{0}\right)^{2} \geq \frac{\zeta^{2}}{4}\end{cases}
$$

Also, let us define a continuous map $T: D \rightarrow X_{v}\left(\mathbb{R}^{N}\right)$ as

$$
T(\tau, \delta)=\tau u^{+}+\delta u^{-}+\zeta \Phi(\tau, \delta) \bar{\sigma} \quad \text { for all }(\tau, \delta) \in D
$$

and $Q: D \rightarrow \mathbb{R}^{2}$ as

$$
Q(\tau, \delta)=\left(\left\langle\mathcal{L}_{\lambda}^{\prime}(T(\tau, \delta)), T(\tau, \delta)^{+}\right\rangle,\left\langle\mathcal{L}_{\lambda}^{\prime}(T(\tau, \delta)), T(\tau, \delta)^{-}\right\rangle\right) \quad \text { for all }(\tau, \delta) \in D .
$$

As the mapping $u \mapsto u^{+}$is continuous in $X_{v}\left(\mathbb{R}^{N}\right)$, we get that $Q$ is also continuous. Furthermore, if we are on the boundary of $D$, that is, $\left(\tau-\tau_{0}\right)^{2}+\left(\delta-\delta_{0}\right)^{2}=\zeta^{2}$, then $\Phi=0$ according to the definition. Therefore, we get $T(\tau, \delta)=\tau u^{+}+\delta u^{-}$and by Lemma 4.1, we deduce 


$$
Q(\tau, \delta) \neq 0 \quad \text { on } \partial D .
$$

Hence, the Brouwer degree is well defined and $\operatorname{deg}(Q, \operatorname{int}(D),(0,0))=1$ and there exists $\left(\tau_{1}, \delta_{1}\right) \in \operatorname{int}(D)$ such that $Q\left(\tau_{1}, \delta_{1}\right)=(0,0)$. Therefore, we get that $T\left(\tau_{1}, \delta_{1}\right) \in \overline{\mathcal{N}}_{\lambda}$ and by the definition of $\overline{d_{\lambda}}$ we deduce that

$$
\mathcal{L}_{\lambda}\left(T\left(\tau_{1}, \delta_{1}\right)\right) \geq \overline{d_{\lambda}}
$$

Next, by equation (40), we have

$$
\begin{aligned}
\mathcal{L}_{\lambda}\left(T\left(\tau_{1}, \delta_{1}\right)\right) & =\mathcal{L}_{\lambda}\left(\tau_{1} u^{+}+\delta_{1} u^{-}\right)+\int_{0}^{1} \frac{d}{d t} \mathcal{L}_{\lambda}\left(\tau_{1} u^{+}+\delta_{1} u^{-}+\zeta t \Phi\left(\tau_{1}, \delta_{1}\right) \bar{\sigma}\right) d t \\
& =\mathcal{L}_{\lambda}\left(\tau_{1} u^{+}+\delta_{1} u^{-}\right)-\zeta \Phi\left(\tau_{1}, \delta_{1}\right) .
\end{aligned}
$$

Now, by definition of $\Phi$ we have $\Phi\left(\tau_{1}, \theta_{1}\right)=1$ when $\left(\tau_{1}, \delta_{1}\right)=\left(\tau_{0}, \delta_{0}\right)$. Hence, we deduce that

$$
\mathcal{L}_{\lambda}\left(T\left(\tau_{1}, \delta_{1}\right)\right) \leq \mathcal{L}_{\lambda}\left(\tau_{1} u^{+}+\delta_{1} u^{-}\right)-\zeta \leq \overline{d_{\lambda}}-\zeta<\overline{d_{\lambda}} .
$$

The case when $\left(\tau_{1}, \delta_{1}\right) \neq\left(\tau_{0}, \delta_{0}\right)$, then by Lemma 4.1 we have

$$
\mathcal{L}_{\lambda}\left(\tau_{1} u^{+}+\delta_{1} u^{-}\right)<\mathcal{L}_{\lambda}\left(\tau_{0} u^{+}+\delta_{0} u^{-}\right)=\overline{d_{\lambda}},
$$

which further gives

$$
\mathcal{L}_{\lambda}\left(T\left(\tau_{1}, \delta_{1}\right)\right) \leq \mathcal{L}_{\lambda}\left(\tau_{1} u^{+}+\delta_{1} u^{-}\right)<\overline{d_{\lambda}} .
$$

This contradicts Eq. (41) and with this we conclude our proof.

Funding Open Access funding provided by the IReL Consortium.

Open Access This article is licensed under a Creative Commons Attribution 4.0 International License, which permits use, sharing, adaptation, distribution and reproduction in any medium or format, as long as you give appropriate credit to the original author(s) and the source, provide a link to the Creative Commons licence, and indicate if changes were made. The images or other third party material in this article are included in the article's Creative Commons licence, unless indicated otherwise in a credit line to the material. If material is not included in the article's Creative Commons licence and your intended use is not permitted by statutory regulation or exceeds the permitted use, you will need to obtain permission directly from the copyright holder. To view a copy of this licence, visit http://creativecommons.org/licenses/by/4.0/.

\section{References}

1. Alves, C.O., Figueiredo, G.M., Yang, M.: Existence of solutions for a nonlinear Choquard equation with potential vanishing at infinity. Adv. Nonlinear Anal. 5(4), 331-345 (2016)

2. Ambrosetti, A., Arcoya, D.: An Introduction to Nonlinear Functional Analysis and Elliptic Problems, Progress in Nonlinear Differential Equations and their Applications, vol. 82. Birkhäuser Boston Inc, Boston (2011)

3. Alves, C.O., Gao, F., Squassina, M., Yang, M.: Singularly perturbed critical Choquard equations. J. Differ. Equ. 263(7), 3943-3988 (2017)

4. Arcoya, D., Boccardo, L.: Multiplicity of solutions for a Dirichlet problem with a singular and a supercritical nonlinearities. Differ. Integral Equ. 26(1-2), 119-128 (2013) 
5. Arcoya, D., Moreno-Mérida, L.: Multiplicity of solutions for a Dirichlet problem with a strongly singular nonlinearity. Nonlinear Anal. 95(1-2), 281-291 (2014)

6. Bal, K., Garain, P.: Multiplicity results for a quasilinear equation with singular nonlinearity. Mediterr. J. Math. 17(100), (2020). https://doi.org/10.1007/s00009-020-01523-5

7. Benhamida, A., Yazidi, H.: Solutions of a weighted $p$-Laplacian critical Sobolev problem. J. Math. Anal. Appl. 487, 123926 (2020)

8. Boccardo, L.: A Dirichlet problem with singular and supercritical nonlinearities. Nonlinear Anal. 75, 4436-4440 (2012)

9. Boccardo, L., Orsina, L.: Semilinear elliptic equations with singular nonlinearities. Calc. Var. Partial Differ. Equ. 37(3-4), 363-380 (2010)

10. Bogachev, V.I.: Measure Theory, vol. I, II. Springer, Berlin (2007)

11. Brezis, H., Nirenberg, L.: Positive solutions of nonlinear elliptic equations involving critical Sobolev exponents. Commun. Pure Appl. Math. 4(36), 437-477 (1983)

12. Cerami, G., Molle, R.: Positive bound state solutions for some Schrödinger-Poisson systems. Nonlinearity 29(10), 3103-3119 (2016)

13. Cerami, G., Vaira, G.: Positive solutions for some non-autonomous Schrödinger-Poisson systems. J. Differ. Equ. 248(3), 521-543 (2010)

14. Chanillo, S., Wheeden, R.L.: Weighted Poincaré and Sobolev inequalities and estimates for weighted Peano maximal functions. Am. J. Math. 107(5), 1191-1226 (1985)

15. Chen, C., Wang, H.: Ground state solutions of singular $p$-Laplacian equation in $\mathbb{R}^{N}$. J. Math. Anal. Appl. 351, 773-780 (2009)

16. Chua, S.-K., Rodney, S., Wheeden, R.: A compact embedding theorem for generalized Sobolev spaces. Pacific J. Math. 265(1), 17-57 (2013)

17. Du, L., Gao, F., Yang, M.: Existence and qualitative analysis for nonlinear weighted Choquard eqautions, arXiv e-prints, arXiv:1810.1175, (2018)

18. De Cicco, V., Vivaldi, M.A.: Weighted Poincaré and Sobolev inequalities and estimates for weighted Peano maximal functions. Adv. Math. Sci. Appl. 9(1), 183-207 (1999)

19. Drábek, P., Kufner, A., Nicolosi, F.: Quasilinear elliptic equations with degenerations and singularities. In: De Gruyter Series in Nonlinear Analysis and Applications, vol. 5. Walter de Gruyter and Co., Berlin (1997)

20. Fabes, E.B., Kenig, C.E., Serapioni, R.P.: The local regularity of solutions of degenerate elliptic equations. Commun. Partial Differ. Equ. 7(1), 77-116 (1982)

21. Furtado, M.F., Souza, B.N.: Positive and nodal solutions for an elliptic equation with critical growth. Commun. Contemp. Math. 18(02), 16 (2016)

22. Garain, P.: On a degenerate singular elliptic problem arXiv e-prints, page arXiv: 1803.02102, (2018)

23. Ghergu, M., Taliaferro, S.D.: Pointwise bounds and blow-up for Choquard-Pekar inequalities at an isolated singularity. J. Differ. Equ. 261(1), 189-217 (2016)

24. Hadiji, R., Yazidi, H.: Problem with critical Sobolev exponent and with weight. Chin. Ann. Math. Ser. B 3(28), 327-352 (2007)

25. Hadiji, R., Molle, R., Passaseo, D., Yazidi, H.: Localization of solutions for nonlinear elliptic problems with weight. C. R. Acad. Sci. Paris, Séc. I Math. 334, 725-730 (2006)

26. Kawohl, B., Lucia, M., Prashanth, S.: Simplicity of the principal eigenvalue for indefinite quasilinear problems. Adv. Differ. Equ. 12, 407-434 (2007)

27. Lions, P.L.: The concentration-compactness principle in the calculus of variations. The locally compact case. II. Ann. Inst. H. Poincaré Anal. Non Linéaire 1(4), 223-283 (1984)

28. Moroz, V., Van Schaftingen, J.: Groundstates of nonlinear Choquard equations: existence, qualitative properties and decay asymptotics. J. Funct. Anal. 265(2), 153-184 (2013)

29. Moroz, V., Van Schaftingen, J.: A guide to the Choquard equation. J. Fixed Point Theory Appl. 19(1), 773-813 (2017)

30. Muckenhoupt, B.: Weighted norm inequalities for the Hardy maximal function. Trans. Am. Math. Soc. 165, 207-226 (1972)

31. Mukherjee, T., Sreenadh, K.: Positive solutions for nonlinear Choquard equation with singular nonlinearity. Complex Var. Elliptic Equ. 62(8), 1044-1071 (2017)

32. Pekar, S.: Untersuchung über die Elektronentheorie der Kristalle. Akademie, Berlin (1954)

33. Penrose, R.: On gravity's role in quantum state reduction. Gen. Relativ. Gravitat. 28(5), 581-600 (1996)

34. Penrose, R.: Quantum computation, entanglement and state reduction. R. Soc. Land. Philos. Trans. Ser. A Math. Phys. Eng. Sci. 3561743, 1927-1939 (1998)

35. Stein, E.M., Weiss, G.: Fractional integrals in n-dimensional Euclidean space. J. Math. Mech. 7, 503514 (1958) 
36. Van Schaftingen, J., Xia, J.: Choquard equations under confining external potentials. NoDEA Nonlinear Differ. Equ. Appl. 24(1), Paper no. 1 (2017)

Publisher's Note Springer Nature remains neutral with regard to jurisdictional claims in published maps and institutional affiliations. 